\title{
New Pieri Type Formulas for Jack Polynomials and their Applications to Interpolation Jack Polynomials
}

\section{Genki SHIBUKAWA}

Department of Mathematics, Kobe University, Rokko, Kobe 657-8501, Japan

E-mail: g-shibukawa@math.kobe-u.ac.jp

Received April 29, 2020, in final form November 14, 2020; Published online November 21, 2020

https://doi.org/10.3842/SIGMA.2020.118

\begin{abstract}
We present new Pieri type formulas for Jack polynomials. As an application, we give a new derivation of higher order difference equations for interpolation Jack polynomials originally found by Knop and Sahi. We also propose Pieri formulas for interpolation Jack polynomials and intertwining relations for a kernel function for Jack polynomials.
\end{abstract}

Key words: Jack polynomial; interpolation Jack polynomial; Pieri formula; kernel function 2020 Mathematics Subject Classification: 05E05; 33C67; 43A90

Dedicated to M. Noumi for his 65th birthday.

\section{Introduction}

Given a positive integer $r$ and a non-zero complex parameter $d$, we define a second-order differential operator in the variables $\mathbf{z}=\left(z_{1}, \ldots, z_{r}\right)$ by

$$
D(\mathbf{z}):=\sum_{j=1}^{r} z_{j}^{2} \partial_{z_{j}}^{2}+d \sum_{1 \leq j \neq l \leq r} \frac{z_{j}^{2}}{z_{j}-z_{l}} \partial_{z_{j}}
$$

where $\partial_{z_{j}}:=\frac{\partial}{\partial z_{j}}$ for $j=1, \ldots, r$. The Jack polynomials are a family of homogeneous symmetric polynomials $P_{\mathbf{m}}\left(\mathbf{z} ; \frac{d}{2}\right)$, indexed by partitions $\mathbf{m}=\left(m_{1}, \ldots, m_{r}\right)$, which are characterized by the differential equation

$$
D(\mathbf{z}) P_{\mathbf{m}}\left(\mathbf{z} ; \frac{d}{2}\right)=P_{\mathbf{m}}\left(\mathbf{z} ; \frac{d}{2}\right) \sum_{j=1}^{r} m_{j}\left(m_{j}-1+d(r-j)\right),
$$

together with the triangularity

$$
P_{\mathbf{m}}\left(\mathbf{z} ; \frac{d}{2}\right)=\sum_{\mathbf{k} \leq \mathbf{m}} c_{\mathbf{m k}} m_{\mathbf{k}}(\mathbf{z}), \quad c_{\mathbf{m k}} \in \mathbb{Q}(d), \quad c_{\mathbf{m m}}=1
$$

with respect to the dominance order on partitions $\mathbf{m}=\left(m_{1}, \ldots, m_{r}\right)$ of a fixed weight $|\mathbf{m}|:=$ $m_{1}+\cdots+m_{r}$

$$
\mathbf{k} \leq \mathbf{m} \Longleftrightarrow \sum_{l=1}^{i} k_{l} \leq \sum_{l=1}^{i} m_{l}, \quad i=1, \ldots, r
$$

This paper is a contribution to the Special Issue on Elliptic Integrable Systems, Special Functions and Quantum Field Theory. The full collection is available at https://www.emis.de/journals/SIGMA/elliptic-integrablesystems.html 
and the monomial symmetric polynomials $m_{\mathbf{m}}(\mathbf{z}):=\sum_{\mathbf{n} \in \mathfrak{S}_{r} \cdot \mathbf{m}} z^{\mathbf{n}}$, where $\mathfrak{S}_{r}$ denotes the symmetric group of degree $r$. Since Jack polynomials $P_{\mathbf{m}}\left(\mathbf{z} ; \frac{d}{2}\right)$ are orthogonal polynomials associated with the root system $A_{r-1}$ and eigenstates of the Calogero-Surtherland model, they play important roles in various fields of mathematics and physics. For their fundamental properties and applications, see $[7,16,17]$.

The interpolation Jack polynomials (or shifted Jack polynomials) are also a family of symmetric polynomials indexed by partitions $\mathbf{m}$, which we denote by $P_{\mathbf{m}}^{\mathrm{ip}}\left(\mathbf{z} ; \frac{d}{2}\right)$ according to the notation of Koornwinder [4]. They are uniquely defined by the following two conditions:

$$
\begin{aligned}
& P_{\mathbf{k}}^{\text {ip }}\left(\mathbf{m}+\frac{d}{2} \boldsymbol{\delta} ; \frac{d}{2}\right)=0, \quad \text { unless } \mathbf{k} \subseteq \mathbf{m} \in \mathcal{P}, \\
& P_{\mathbf{m}}^{\text {ip }}\left(\mathbf{z} ; \frac{d}{2}\right)=P_{\mathbf{m}}\left(\mathbf{z} ; \frac{d}{2}\right)+(\text { lower degree terms }),
\end{aligned}
$$

where $\boldsymbol{\delta}$ denotes the staircase partition $(r-1, r-2, \ldots, 2,1,0)$ and $\mathbf{k} \subseteq \mathbf{m}$ stands for the inclusion partial order defined by

$$
\mathbf{k} \subseteq \mathbf{m} \quad \Longleftrightarrow \quad k_{i} \leq m_{i}, \quad i=1, \ldots, r .
$$

These polynomials $P_{\mathbf{m}}^{\mathrm{ip}}\left(\mathbf{z} ; \frac{d}{2}\right)$ were introduced by Sahi [13], Knop-Sahi [3] and OkounkovOlshanski [10] as a continuous deformation of shifted Schur polynomials $P_{\mathbf{m}}^{\mathrm{ip}}(\mathbf{z} ; 1)$ [6,11]. The interpolation Jack polynomials appear as a multivariate analogue of the falling factorials

$$
P_{m}^{\mathrm{ip}}(z):= \begin{cases}z(z-1) \cdots(z-m+1), & m \neq 0 \\ 1, & m=0\end{cases}
$$

in explicit formulas of binomial type for multivariate hypergeometric functions (see $[4,8,9])$.

We review some fundamental results on Jack and interpolation Jack polynomials relevant to the main results of this paper.

1) Sekiguchi operators (Debiard [2], Macdonald [7], Sekiguchi [15]). The Jack polynomials are joint eigenfunctions of a commuting family of differential operators (Sekiguchi operators). We define Sekiguchi operators $H_{r, p}^{(d)}(\mathbf{z})(p=0,1, \ldots, r)$ and their generating function $S_{r}^{(d)}(u ; \mathbf{z})$ by

$$
\begin{aligned}
& H_{r, p}^{(d)}(\mathbf{z}):=\sum_{l=0}^{p}\left(\frac{2}{d}\right)^{p-l} \sum_{\substack{I \subseteq[r],|\bar{I}|=l}}\left(\frac{1}{\Delta(\mathbf{z})}\left(\prod_{i \in I} z_{i} \partial_{z_{i}}\right) \Delta(\mathbf{z})\right) \sum_{\substack{J \subseteq[r] \backslash I \\
|J|=p-l}}\left(\prod_{j \in J} z_{j} \partial_{z_{j}}\right), \\
& S_{r}^{(d)}(u ; \mathbf{z}):=\sum_{p=0}^{r} H_{r, p}^{(d)}(\mathbf{z}) u^{r-p}
\end{aligned}
$$

respectively, where $[r]:=\{1,2, \ldots, r\}$ and $\Delta(\mathbf{z}):=\prod_{1 \leq i<j \leq r}\left(z_{i}-z_{j}\right)$. Then we have

$$
\begin{aligned}
& H_{r, p}^{(d)}(\mathbf{z}) P_{\mathbf{m}}\left(\mathbf{z} ; \frac{d}{2}\right)=P_{\mathbf{m}}\left(\mathbf{z} ; \frac{d}{2}\right) e_{r, p}\left(\mathbf{m}+\frac{d}{2} \boldsymbol{\delta}\right), \\
& S_{r}^{(d)}(u ; \mathbf{z}) P_{\mathbf{m}}\left(\mathbf{z} ; \frac{d}{2}\right)=P_{\mathbf{m}}\left(\mathbf{z} ; \frac{d}{2}\right) I_{r}^{(d)}(u ; \mathbf{m}),
\end{aligned}
$$

where

$$
e_{r, k}(\mathbf{z}):=\sum_{1 \leq i_{1}<\cdots<i_{k} \leq r} z_{i_{1}} \cdots z_{i_{k}}, \quad k=1, \ldots, r, \quad e_{r, 0}(\mathbf{z}):=1,
$$




$$
I_{r}^{(d)}(u ; \mathbf{m}):=\prod_{k=1}^{r}\left(u+r-k+\frac{2}{d} m_{k}\right)=\left(\frac{2}{d}\right)^{r} \prod_{k=1}^{r}\left(m_{k}+\frac{d}{2}(u+r-k)\right) .
$$

2) Pieri type formulas for Jack polynomials (Lassalle [5], Stanley [16]). We introduce two kinds of normalization for Jack polynomials,

$$
\begin{aligned}
\Phi_{\mathbf{m}}^{(d)}(\mathbf{z}) & :=\frac{P_{\mathbf{m}}\left(\mathbf{z} ; \frac{d}{2}\right)}{P_{\mathbf{m}}\left(\mathbf{1} ; \frac{d}{2}\right)}, \\
\Psi_{\mathbf{m}}^{(d)}(\mathbf{z}) & :=\frac{P_{\mathbf{m}}\left(\mathbf{z} ; \frac{d}{2}\right)}{P_{\mathbf{m}}^{\text {ip }}\left(\mathbf{m}+\frac{d}{2} \boldsymbol{\delta} ; \frac{d}{2}\right)}=\frac{P_{\mathbf{m}}\left(\mathbf{1} ; \frac{d}{2}\right)}{P_{\mathbf{m}}^{\mathrm{ip}}\left(\mathbf{m}+\frac{d}{2} \boldsymbol{\delta} ; \frac{d}{2}\right)} \Phi_{\mathbf{m}}^{(d)}(\mathbf{z}),
\end{aligned}
$$

where $\mathbf{1}:=(1, \ldots, 1)$. For the explicit formulas of $P_{\mathbf{m}}\left(\mathbf{1} ; \frac{d}{2}\right)$ and $P_{\mathbf{m}}^{\mathrm{ip}}\left(\mathbf{m}+\frac{d}{2} \boldsymbol{\delta} ; \frac{d}{2}\right)$, we refer to [7] and [4]. We also use the notations

$$
\begin{aligned}
& \mathcal{P}:=\left\{\mathbf{m}=\left(m_{1}, \ldots, m_{r}\right) \in \mathbb{Z}^{r} \mid m_{1} \geq \cdots \geq m_{r} \geq 0\right\}, \\
&|\mathbf{z}|:=\sum_{j=1}^{r} z_{j}, \quad\left|\partial_{\mathbf{z}}\right|:=\sum_{j=1}^{r} \partial_{z_{j}}, \quad A_{ \pm, i}^{(d)}(\mathbf{x}):=\prod_{1 \leq k \neq i \leq r} \frac{x_{i}-x_{k}-\frac{d}{2}(i-k) \pm \frac{d}{2}}{x_{i}-x_{k}-\frac{d}{2}(i-k)}, \\
& \epsilon_{i}:=(0, \ldots, 0, \stackrel{i}{1}, 0, \ldots, 0) \in \mathbb{Z}^{r} .
\end{aligned}
$$

Then the Jack polynomials satisfy two Pieri type formulas ${ }^{1}$ for the differential operator $\left|\partial_{\mathbf{z}}\right|$ [5, equation (14.1)]

$$
\begin{aligned}
\left|\partial_{\mathbf{z}}\right| \Phi_{\mathbf{x}}^{(d)}(\mathbf{z}) & =\sum_{i=1}^{r} \Phi_{\mathbf{x}-\epsilon_{i}}^{(d)}(\mathbf{z})\left(x_{i}+\frac{d}{2}(r-i)\right) A_{-, i}^{(d)}(\mathbf{x}), \\
\left|\partial_{\mathbf{z}}\right| \Psi_{\mathbf{x}}^{(d)}(\mathbf{z}) & =\sum_{\substack{1 \leq i \leq r, \mathbf{x}-\epsilon_{i} \in \mathcal{P}}} \Psi_{\mathbf{x}-\epsilon_{i}}^{(d)}(\mathbf{z}) A_{+, i}^{(d)}\left(\mathbf{x}-\epsilon_{i}\right)
\end{aligned}
$$

and for the multiplication operator $|\mathbf{z}|$ [16, Theorem 6.1] (see also Corollary 5.2 in our article and [7, equation (6.24)])

$$
\begin{aligned}
& |\mathbf{z}| \Phi_{\mathbf{x}}^{(d)}(\mathbf{z})=\sum_{i=1}^{r} \Phi_{\mathbf{x}+\epsilon_{i}}^{(d)}(\mathbf{z}) A_{+, i}^{(d)}(\mathbf{x}) \\
& |\mathbf{z}| \Psi_{\mathbf{x}}^{(d)}(\mathbf{z})=\sum_{\substack{1 \leq i \leq r, \mathbf{x}+\epsilon_{i} \in \mathcal{P}}} \Psi_{\mathbf{x}+\epsilon_{i}}^{(d)}(\mathbf{z})\left(x_{i}+1+\frac{d}{2}(r-i)\right) A_{-, i}^{(d)}\left(\mathbf{x}+\epsilon_{i}\right) .
\end{aligned}
$$

We remark that if $\mathbf{x}-\epsilon_{i} \notin \mathcal{P}\left(\right.$ resp. $\left.\mathbf{x}+\epsilon_{i} \notin \mathcal{P}\right)$ then $A_{-, i}^{(d)}(\mathbf{x})=0\left(\operatorname{resp} . A_{+, i}^{(d)}(\mathbf{x})=0\right)$.

3) Binomial formula (Knop-Sahi [3], Okounkov-Olshanski [10]). For any partition x, Jack polynomials with variables shifted by $\mathbf{1}$ are written in the form

$$
\Phi_{\mathbf{x}}^{(d)}(\mathbf{1}+\mathbf{z})=\sum_{\mathbf{k} \subseteq \mathbf{x}} \frac{P_{\mathbf{k}}^{\mathrm{ip}}\left(\mathbf{x}+\frac{d}{2} \boldsymbol{\delta} ; \frac{d}{2}\right)}{P_{\mathbf{k}}\left(\mathbf{1} ; \frac{d}{2}\right)} \Psi_{\mathbf{k}}^{(d)}(\mathbf{z}) .
$$

\footnotetext{
${ }^{1}$ Although these naming for (1.2) and (1.3) may not be appropriate, we have not been able to give an appropriate name for these formulas and will call them Pieri type formulas in this paper.
} 
In this article, we propose new Pieri type formulas for Jack polynomials that generalize (1.2) and (1.3), which we call the twisted Pieri formulas. They give explicit expressions of the action of the operator

$$
\frac{\left(\operatorname{ad}\left|\partial_{\mathbf{z}}\right|\right)^{l}}{l !} S_{r}^{(d)}(u ; \mathbf{z})
$$

on $\Phi_{\mathbf{x}}^{(d)}(\mathbf{z})$ and $\Psi_{\mathbf{x}}^{(d)}(\mathbf{z})$, where ad denotes the usual commutator defined by $(\operatorname{ad} A)(B):=$ $A B-B A$.

Theorem 1.1 (twisted Pieri formulas for Jack polynomials). For $l=0,1, \ldots, r$, we have

$$
\begin{aligned}
& {\left[\frac{\left(\operatorname{ad}\left|\partial_{\mathbf{z}}\right|\right)^{l}}{l !} S_{r}^{(d)}(u ; \mathbf{z})\right] \Phi_{\mathbf{x}}^{(d)}(\mathbf{z})=\sum_{\substack{J \subseteq[r],|J|=l}} \Phi_{\mathbf{x}-\epsilon_{J}}^{(d)}(\mathbf{z}) I_{J^{c}}^{(d)}(u ; \mathbf{x}) A_{-, J}^{(d)}(\mathbf{x}) \prod_{j \in J}\left(x_{j}+\frac{d}{2}(r-j)\right),} \\
& {\left[\frac{\left(\operatorname{ad}\left|\partial_{\mathbf{z}}\right|\right)^{l}}{l !} S_{r}^{(d)}(u ; \mathbf{z})\right] \Psi_{\mathbf{x}}^{(d)}(\mathbf{z})=\sum_{\substack{J \subseteq[r],|J|=l, \mathbf{x}-\epsilon_{J} \in \mathcal{P}}} \Psi_{\mathbf{x}-\epsilon_{J}}^{(d)}(\mathbf{z}) I_{J^{c}}^{(d)}(u ; \mathbf{x}) A_{+, J}^{(d)}\left(\mathbf{x}-\epsilon_{J}\right),}
\end{aligned}
$$

where $J^{c}:=[r] \backslash J, \epsilon_{J}:=\sum_{j \in J} \epsilon_{j}$ and

$$
\begin{aligned}
& A_{ \pm, J}^{(d)}(\mathbf{x}):=\prod_{j \in J, l \in J^{c}} \frac{x_{j}-x_{l}-\frac{d}{2}(j-l) \pm \frac{d}{2}}{x_{j}-x_{l}-\frac{d}{2}(j-l)}, \\
& I_{J^{c}}^{(d)}(u ; \mathbf{x}):=\left(\frac{2}{d}\right)^{r} \prod_{l \in J^{c}}\left(x_{l}+\frac{d}{2}(u+r-l)\right) .
\end{aligned}
$$

Since the first-order Sekiguchi operator $H_{r, 1}^{(d)}(\mathbf{z})$ coincides with the Euler operator $\sum_{i=1}^{r} z_{i} \partial_{z_{i}}$ essentially

$$
H_{r, 1}^{(d)}(\mathbf{z})=\frac{2}{d} \sum_{i=1}^{r} z_{i} \partial_{z_{i}}+\frac{r(r-1)}{2},
$$

we write down the operator $\left(\operatorname{ad}\left|\partial_{\mathbf{z}}\right|\right) H_{r, 1}^{(d)}(\mathbf{z})$

$$
\left(\operatorname{ad}\left|\partial_{\mathbf{z}}\right|\right) H_{r, 1}^{(d)}(\mathbf{z})=\left(\operatorname{ad}\left|\partial_{\mathbf{z}}\right|\right)\left(\frac{2}{d} \sum_{i=1}^{r} z_{i} \partial_{z_{i}}\right)=\frac{2}{d}\left|\partial_{\mathbf{z}}\right| .
$$

Therefore twisted Pieri formulas (1.6) and (1.7) are regarded as a higher order analogue of the above Pieri type formulas (1.2) and (1.3) respectively. See also Corollary 5.1.

From the twisted Pieri formulas for Jack of Theorem 1.1, we obtain three important results as follows. The first one is an alternative proof of the following theorem on difference equations for interpolation Jack polynomials due to Knop-Sahi [3] (see also Corollary 3.1).

Theorem 1.2 (Knop-Sahi). For any $\mathbf{x} \in \mathbb{C}^{r}$ and $\mathbf{k} \in \mathcal{P}$, we have

$$
D_{r}^{(d) \operatorname{ip}}(u ; \mathbf{x}) P_{\mathbf{k}}^{\text {ip }}\left(\mathbf{x}+\frac{d}{2} \boldsymbol{\delta} ; \frac{d}{2}\right)=P_{\mathbf{k}}^{\text {ip }}\left(\mathbf{x}+\frac{d}{2} \boldsymbol{\delta} ; \frac{d}{2}\right) I_{r}^{(d)}(u ; \mathbf{k}),
$$

where

$$
\begin{aligned}
& D_{r}^{(d) \operatorname{ip}}(u ; \mathbf{x}):=\sum_{J \subseteq[r]}(-1)^{|J|} I_{J^{c}}^{(d)}(u ; \mathbf{x}) A_{-, J}^{(d)}(\mathbf{x}) \prod_{j \in J}\left(x_{j}+\frac{d}{2}(r-j)\right) T_{\mathbf{x}}^{J}, \\
& T_{x_{j}} f(\mathbf{x}):=f\left(\mathbf{x}-\epsilon_{j}\right), \quad T_{\mathbf{x}}^{J}:=\prod_{j \in J} T_{x_{j}} .
\end{aligned}
$$


The second result is the Pieri formulas for interpolation Jack polynomials (see also Corollary 4.1).

Theorem 1.3. For any $\mathbf{x} \in \mathbb{C}^{r}$ and $\mathbf{k} \in \mathcal{P}$, we have

$$
I_{r}^{(d)}(u ; \mathbf{x}) \frac{P_{\mathbf{k}}^{\mathrm{ip}}\left(\mathbf{x}+\frac{d}{2} \boldsymbol{\delta} ; \frac{d}{2}\right)}{P_{\mathbf{k}}\left(\mathbf{1} ; \frac{d}{2}\right)}=\sum_{\substack{J \subseteq[r], \mathbf{k}+\epsilon_{J} \in \mathcal{P}}} \frac{P_{\mathbf{k}+\epsilon_{J}}^{\mathrm{ip}}\left(\mathbf{x}+\frac{d}{2} \boldsymbol{\delta} ; \frac{d}{2}\right)}{P_{\mathbf{k}+\epsilon_{J}}\left(\mathbf{1} ; \frac{d}{2}\right)} I_{J^{c}}^{(d)}(u ; \mathbf{k}) A_{+, J}^{(d)}(\mathbf{k}) .
$$

Finally, we obtain the following intertwining relation for a kernel function of Jack polynomials $[5,17]$

$$
{ }_{0} \mathcal{F}_{0}{ }^{(d)}(\mathbf{z}, \mathbf{w}):=\sum_{\mathbf{m} \in \mathcal{P}} \Psi_{\mathbf{m}}^{(d)}(\mathbf{z}) \Phi_{\mathbf{m}}^{(d)}(\mathbf{w})=\sum_{\mathbf{m} \in \mathcal{P}} \Phi_{\mathbf{m}}^{(d)}(\mathbf{z}) \Psi_{\mathbf{m}}^{(d)}(\mathbf{w}),
$$

which is a multivariate analogue of

$$
e^{z w}=\sum_{m=0}^{\infty} \frac{1}{m !} z^{m} w^{m}=\sum_{m=0}^{\infty} \Psi_{m}(z) \Phi_{m}(w)=\sum_{m=0}^{\infty} \Phi_{m}(z) \Psi_{m}(w) .
$$

Theorem 1.4. For any $l=0,1, \ldots, r$, we have

$$
\left(\frac{d}{2}\right)^{l}\left[\frac{\left(\operatorname{ad}\left|\partial_{\mathbf{z}}\right|\right)^{l}}{l !} H_{r, l}^{(d)}(\mathbf{z})\right]{ }_{0} \mathcal{F}_{0}{ }^{(d)}(\mathbf{z}, \mathbf{w})={ }_{0} \mathcal{F}_{0}{ }^{(d)}(\mathbf{z}, \mathbf{w}) e_{r, l}(\mathbf{w}) .
$$

It is a multivariate analogue of the relation

$$
\partial_{z} e^{z w}=e^{z w} w
$$

and a higher order analogue of the formula in [5] Section 14

$$
\left|\partial_{\mathbf{z}}\right|_{0} \mathcal{F}_{0}^{(d)}(\mathbf{z}, \mathbf{w})={ }_{0} \mathcal{F}_{0}^{(d)}(\mathbf{z}, \mathbf{w})|\mathbf{w}| .
$$

The contents of this article are as follows. In Section 2, we prove the twisted Pieri formulas for Jack polynomials. From the twisted Pieri formulas for Jack polynomials, we give another proof of Theorem 1.2 in Section 3 and prove Theorem 1.3 in Section 4. We also prove the intertwining relation (1.10) for the kernel function ${ }_{0} \mathcal{F}_{0}{ }^{(d)}(\mathbf{z}, \mathbf{w})$ in Section 5 . Finally, we mention some future works for twisted Pieri formulas and their applications in Section 6.

\section{Twisted Pieri formulas for Jack polynomials}

To prove Theorem 1.1, we need the following summation formula.

Lemma 1 (mysterious summation). For any $I \subseteq[r]$ and $\mathbf{x}=\left(x_{1}, \ldots, x_{r}\right) \in \mathcal{P}$, we have

$$
\begin{aligned}
\sum_{i \in I}( & \left.x_{i}+1+\frac{d}{2}(r-i)\right) A_{-, i, I \backslash i}^{(d)}\left(\mathbf{x}+\epsilon_{i}\right) A_{+, i, I \backslash i}^{(d)}(\mathbf{x}) \\
& -\sum_{i \in I}\left(x_{i}+\frac{d}{2}(r-i)\right) A_{+, i, I \backslash i}^{(d)}\left(\mathbf{x}-\epsilon_{i}\right) A_{-, i, I \backslash i}^{(d)}(\mathbf{x})=|I|,
\end{aligned}
$$

where $|I|$ is the cardinality of $I$ and

$$
A_{ \pm, i, I \backslash i}^{(d)}(\mathbf{x}):=\prod_{j \in I \backslash i} \frac{x_{i}-x_{j}-\frac{d}{2}(i-j) \pm \frac{d}{2}}{x_{i}-x_{j}-\frac{d}{2}(i-j)} .
$$


If $r=1$, then (2.1) is equal to a trivial summation

$$
(x+1)-x=1 .
$$

Proof. For convenience, we put

$$
s_{j}:=x_{j}+\frac{d}{2}(r-j) .
$$

By Pieri type formulas for the Jack polynomials (1.2) and (1.4), we have

$$
\begin{aligned}
& {\left[\left|\partial_{\mathbf{z}}\right|,|\mathbf{z}|\right] \Phi_{\mathbf{x}}^{(d)}(\mathbf{z})} \\
& \quad=\sum_{i=1}^{r}\left|\partial_{\mathbf{z}}\right| \Phi_{\mathbf{x}+\epsilon_{i}}^{(d)}(\mathbf{z}) A_{+, i}^{(d)}(\mathbf{x})-\sum_{j=1}^{r}|\mathbf{z}| \Phi_{\mathbf{x}-\epsilon_{j}}^{(d)}(\mathbf{z}) s_{j} A_{-, j}^{(d)}(\mathbf{x}) \\
& \quad=\sum_{i=1}^{r} \sum_{j=1}^{r} \Phi_{\mathbf{x}+\epsilon_{i}-\epsilon_{j}}^{(d)}(\mathbf{z})\left(\left(s_{j}+\delta_{j, i}\right) A_{-, j}^{(d)}\left(\mathbf{x}+\epsilon_{i}\right) A_{+, i}^{(d)}(\mathbf{x})-s_{j} A_{-, j}^{(d)}(\mathbf{x}) A_{+, i}^{(d)}\left(\mathbf{x}-\epsilon_{j}\right)\right) \\
& =\Phi_{\mathbf{x}}^{(d)}(\mathbf{z}) \sum_{i=1}^{r}\left(\left(s_{i}+1\right) A_{-, i}^{(d)}\left(\mathbf{x}+\epsilon_{i}\right) A_{+, i}^{(d)}(\mathbf{x})-s_{i} A_{-, i}^{(d)}(\mathbf{x}) A_{+, i}^{(d)}\left(\mathbf{x}-\epsilon_{i}\right)\right) .
\end{aligned}
$$

On the other hand,

$$
\left[\left|\partial_{\mathbf{z}}\right|,|\mathbf{z}|\right] \Phi_{\mathbf{x}}^{(d)}(\mathbf{z})=\Phi_{\mathbf{x}}^{(d)}(\mathbf{z}) r .
$$

Then, we obtain (2.1).

Proof of Theorem 1.1. Since (1.6) and (1.7) can be similarly proved, we only prove (1.6). These formulas are proved by induction on $l$.

The case of $l=0$ is

$$
S_{r}^{(d)}(u ; \mathbf{z}) \Phi_{\mathbf{x}}^{(d)}(\mathbf{z})=\Phi_{\mathbf{x}}^{(d)}(\mathbf{z}) I_{r}^{(d)}(u ; \mathbf{x}) .
$$

This is $(1.1)$

$$
S_{r}^{(d)}(u ; \mathbf{z}) P_{\mathbf{m}}\left(\mathbf{z} ; \frac{d}{2}\right)=P_{\mathbf{m}}\left(\mathbf{z} ; \frac{d}{2}\right) I_{r}^{(d)}(u ; \mathbf{m})
$$

exactly.

If $l=1$, then

$$
\begin{aligned}
& {\left[\left(\operatorname{ad}\left|\partial_{\mathbf{z}}\right|\right) S_{r}^{(d)}(u ; \mathbf{z})\right] \Phi_{\mathbf{x}}^{(d)}(\mathbf{z})} \\
& \quad=\left|\partial_{\mathbf{z}}\right| \Phi_{\mathbf{x}}^{(d)}(\mathbf{z}) I_{r}^{(d)}(u ; \mathbf{x})-S_{r}^{(d)}(u ; \mathbf{z}) \sum_{i=1}^{r} \Phi_{\mathbf{x}-\epsilon_{i}}^{(d)}(\mathbf{z})\left(x_{i}+\frac{d}{2}(r-i)\right) A_{-, i}^{(d)}(\mathbf{x}) \\
& \quad=\sum_{i=1}^{r} \Phi_{\mathbf{x}-\epsilon_{i}}^{(d)}(\mathbf{z}) s_{i} A_{-, i}^{(d)}(\mathbf{x})\left(I_{r}^{(d)}(u ; \mathbf{x})-I_{r}^{(d)}\left(u ; \mathbf{x}-\epsilon_{i}\right)\right) \\
& =\sum_{\substack{J \subseteq[r] \\
|\bar{J}|=1}} \Phi_{\mathbf{x}-\epsilon_{J}}^{(d)}(\mathbf{z}) I_{J^{c}}^{(d)}(u ; \mathbf{x}) A_{-, J}^{(d)}(\mathbf{x}) s_{i} .
\end{aligned}
$$

Here, the first and second equalities follow from (1.1) and (1.2) respectively.

Assume the $n=l$ case holds. Hence, from the induction hypothesis and (1.2) we have

$$
\left[\frac{\left(\operatorname{ad}\left|\partial_{\mathbf{z}}\right|\right)^{l+1}}{(l+1) !} S_{r}^{(d)}(u ; \mathbf{z})\right] \Phi_{\mathbf{x}}^{(d)}(\mathbf{z})
$$




$$
\begin{aligned}
& =\frac{1}{l+1}\left|\partial_{\mathbf{z}}\right|\left[\frac{\left(\operatorname{ad}\left|\partial_{\mathbf{z}}\right|\right)^{l}}{l !} S_{r}^{(d)}(u ; \mathbf{z})\right] \Phi_{\mathbf{x}}^{(d)}(\mathbf{z})-\left[\frac{\left(\operatorname{ad}\left|\partial_{\mathbf{z}}\right|\right)^{l}}{l !} S_{r}^{(d)}(u ; \mathbf{z})\right] \frac{1}{l+1}\left|\partial_{\mathbf{z}}\right| \Phi_{\mathbf{x}}^{(d)}(\mathbf{z})
\end{aligned}
$$

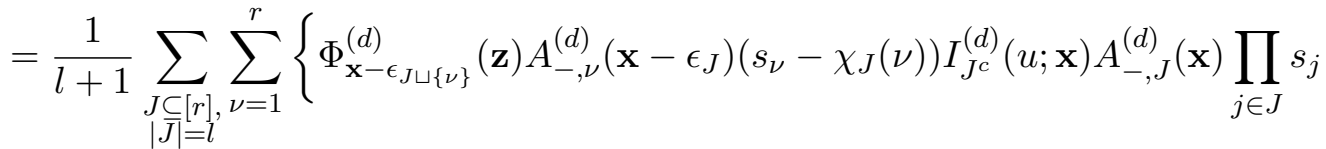

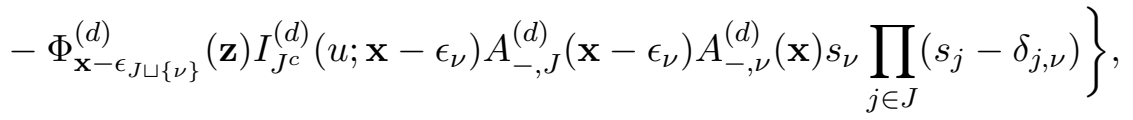

where $\delta_{j, \nu}$ is the Kronecker's delta and

$$
\chi_{J}(\nu):= \begin{cases}1, & \nu \in J \\ 0, & \nu \notin J\end{cases}
$$

From a simple calculation, we have

$$
\begin{aligned}
{\left[\frac{\left(\operatorname{ad}\left|\partial_{\mathbf{z}}\right|\right)^{l+1}}{(l+1) !} S_{r}^{(d)}(u ; \mathbf{z})\right] \Phi_{\mathbf{x}}^{(d)}(\mathbf{z})=} & \sum_{\substack{I \subseteq[r],|I|=l+1}} \frac{1}{l+1} \Phi_{\mathbf{x}-\epsilon_{I}}^{(d)}(\mathbf{z}) I_{I^{c}}^{(d)}(u ; \mathbf{x}) A_{-, I}^{(d)}(\mathbf{x}) \prod_{j \in I} s_{j} \\
& \times \sum_{i \in I}\left\{\left(s_{i}+\frac{d}{2} u\right) A_{-,\{i\}, I \backslash\{i\}}^{(d)}\left(\mathbf{x}+\epsilon_{i}\right) A_{+,\{i\}, I \backslash\{i\}}^{(d)}(\mathbf{x})\right. \\
& \left.-\left(s_{i}-1+\frac{d}{2} u\right) A_{-,\{i\}, I \backslash\{i\}}^{(d)}(\mathbf{x}) A_{+,\{i\}, I \backslash\{i\}}^{(d)}\left(\mathbf{x}-\epsilon_{i}\right)\right\} .
\end{aligned}
$$

Since the summation

$$
\begin{aligned}
\sum_{i \in I}\{ & \left.s_{i} A_{-,\{i\}, I \backslash\{i\}}^{(d)}\left(\mathbf{x}+\epsilon_{i}\right) A_{+,\{i\}, I \backslash\{i\}}^{(d)}(\mathbf{x})-\left(s_{i}-1\right) A_{-,\{i\}, I \backslash\{i\}}^{(d)}(\mathbf{x}) A_{+,\{i\}, I \backslash\{i\}}^{(d)}\left(\mathbf{x}-\epsilon_{i}\right)\right\} \\
& =l+1
\end{aligned}
$$

is our mysterious summation (2.1) exactly, we obtain the conclusion (1.6).

\section{Another proof of difference equations for interpolation Jack polynomials}

Proof of Theorem 1.2. Since the difference equation (1.8) is a relation for rational function of $\left(x_{1}, \ldots, x_{r}\right)$, it is enough to prove (1.8) for any partition $\mathbf{x} \in \mathcal{P}$. To prove (1.8), we compute

$$
S_{r}^{(d)}(u ; \mathbf{z}) \Phi_{\mathbf{x}}^{(d)}(\mathbf{1}+\mathbf{z})
$$

in two different ways. First, a simple calculation shows that

$$
\begin{aligned}
S_{r}^{(d)}(u ; \mathbf{z}) \Phi_{\mathbf{x}}^{(d)}(\mathbf{1}+\mathbf{z}) & =S_{r}^{(d)}(u ; \mathbf{z}) e^{\left|\partial_{\mathbf{z}}\right|} \Phi_{\mathbf{x}}^{(d)}(\mathbf{z})=e^{\left|\partial_{\mathbf{z}}\right|} e^{-\left|\partial_{\mathbf{z}}\right|} S_{r}^{(d)}(u ; \mathbf{z}) e^{\left|\partial_{\mathbf{z}}\right|} \Phi_{\mathbf{x}}^{(d)}(\mathbf{z}) \\
& =e^{\left|\partial_{\mathbf{z}}\right|}\left[e^{-\operatorname{ad}\left|\partial_{\mathbf{z}}\right|} S_{r}^{(d)}(u ; \mathbf{z})\right] \Phi_{\mathbf{x}}^{(d)}(\mathbf{z}) .
\end{aligned}
$$

Since the highest derivative in $H_{r, p}^{(d)}(\mathbf{z})$ has degree $p$, the sum

$$
e^{-\operatorname{ad}\left|\partial_{\mathbf{z}}\right|} S_{r}^{(d)}(u ; \mathbf{z})=\sum_{l \geq 0} \frac{\left(-\operatorname{ad}\left|\partial_{\mathbf{z}}\right|\right)^{l}}{l !} S_{r}^{(d)}(u ; \mathbf{z})
$$


terminates after $\left(-\operatorname{ad}\left|\partial_{\mathbf{z}}\right|\right)^{r}$. Then, we have

$$
S_{r}^{(d)}(u ; \mathbf{z}) \Phi_{\mathbf{x}}^{(d)}(\mathbf{1}+\mathbf{z})=e^{\left|\partial_{\mathbf{z}}\right|} \sum_{l=0}^{r}\left[\frac{\left(-\operatorname{ad}\left|\partial_{\mathbf{z}}\right|\right)^{l}}{l !} S_{r}^{(d)}(u ; \mathbf{z})\right] \Phi_{\mathbf{x}}^{(d)}(\mathbf{z}) .
$$

By applying the twisted Pieri (1.6) and the binomial (1.5), we have

$$
\begin{aligned}
S_{r}^{(d)}(u ; \mathbf{z}) \Phi_{\mathbf{x}}^{(d)}(\mathbf{1}+\mathbf{z})= & \sum_{l=0}^{r}(-1)^{l} \sum_{\substack{J \subseteq[r] \\
|J|=l}} e^{\left|\partial_{\mathbf{z}}\right|} \Phi_{\mathbf{x}-\epsilon_{J}}^{(d)}(\mathbf{z}) I_{J^{c}}^{(d)}(u ; \mathbf{x}) A_{-, J}^{(d)}(\mathbf{x}) \prod_{j \in J}\left(x_{j}+\frac{d}{2}(r-j)\right) \\
= & \sum_{J \subseteq[r]}(-1)^{|J|} \Phi_{\mathbf{x}-\epsilon_{J}}^{(d)}(\mathbf{1}+\mathbf{z}) I_{J^{c}}^{(d)}(u ; \mathbf{x}) A_{-, J}^{(d)}(\mathbf{x}) \prod_{j \in J}\left(x_{j}+\frac{d}{2}(r-j)\right) \\
= & \sum_{\mathbf{k} \in \mathcal{P}} \Psi_{\mathbf{k}}^{(d)}(\mathbf{z}) \sum_{J \subseteq[r]}(-1)^{|J|} \frac{P_{\mathbf{k}}^{\mathrm{ip}}\left(\mathbf{x}-\epsilon_{J}+\frac{d}{2} \boldsymbol{\delta} ; \frac{d}{2}\right)}{P_{\mathbf{k}}\left(\mathbf{1} ; \frac{d}{2}\right)} I_{J^{c}}^{(d)}(u ; \mathbf{x}) \\
& \times A_{-, J}^{(d)}(\mathbf{x}) \prod_{j \in J}\left(x_{j}+\frac{d}{2}(r-j)\right) .
\end{aligned}
$$

On the other hand, from the binomial formula (1.5) and (1.1), we have

$$
\begin{aligned}
S_{r}^{(d)}(u ; \mathbf{z}) \Phi_{\mathbf{x}}^{(d)}(\mathbf{1}+\mathbf{z}) & =\sum_{\mathbf{k} \subseteq \mathbf{x}} \frac{P_{\mathbf{k}}^{\mathrm{ip}}\left(\mathbf{x}+\frac{d}{2} \boldsymbol{\delta} ; \frac{d}{2}\right)}{P_{\mathbf{k}}\left(\mathbf{1} ; \frac{d}{2}\right)} S_{r}^{(d)}(u ; \mathbf{z}) \Psi_{\mathbf{k}}^{(d)}(\mathbf{z}) \\
& =\sum_{\mathbf{k} \subseteq \mathbf{x}} \frac{P_{\mathbf{k}}^{\mathrm{ip}}\left(\mathbf{x}+\frac{d}{2} \boldsymbol{\delta} ; \frac{d}{2}\right)}{P_{\mathbf{k}}\left(\mathbf{1} ; \frac{d}{2}\right)} \Psi_{\mathbf{k}}^{(d)}(\mathbf{z}) I_{r}^{(d)}(u ; \mathbf{x}) \\
& =\sum_{\mathbf{k} \in \mathcal{P}} \Psi_{\mathbf{k}}^{(d)}(\mathbf{z}) I_{r}^{(d)}(u ; \mathbf{x}) \frac{P_{\mathbf{k}}^{\text {ip }}\left(\mathbf{x}+\frac{d}{2} \boldsymbol{\delta} ; \frac{d}{2}\right)}{P_{\mathbf{k}}\left(\mathbf{1} ; \frac{d}{2}\right)} .
\end{aligned}
$$

By comparing coefficients for $\Psi_{\mathbf{k}}^{(d)}(\mathbf{z})$ in (3.1) and (3.2), we obtain the conclusion (1.8).

Comparing coefficients for $u^{r-l}$ in (1.8), we obtain higher order difference formulas for interpolation Jack polynomials.

Corollary 3.1. For any $\mathbf{x} \in \mathbb{C}^{r}, \mathbf{k} \in \mathcal{P}$ and $l=0,1, \ldots, r$, we have

$$
\begin{aligned}
e_{r, l}\left(\mathbf{k}+\frac{d}{2} \boldsymbol{\delta}\right) P_{\mathbf{k}}^{\mathrm{ip}}\left(\mathbf{x}+\frac{d}{2} \boldsymbol{\delta} ; \frac{d}{2}\right)= & \sum_{\substack{J \subseteq[r], 0 \leq|J| \leq l}}(-1)^{|J|} P_{\mathbf{k}}^{\mathrm{ip}}\left(\mathbf{x}-\epsilon_{J}+\frac{d}{2} \boldsymbol{\delta} ; \frac{d}{2}\right) \\
& \times e_{r-|J|, l-|J|}\left(\left(\mathbf{x}+\frac{d}{2} \boldsymbol{\delta}\right)_{J^{c}}\right) A_{-, J}^{(d)}(\mathbf{x}) \prod_{j \in J}\left(x_{j}+\frac{d}{2}(r-j)\right),
\end{aligned}
$$

where

$$
\left(\mathbf{x}+\frac{d}{2} \boldsymbol{\delta}\right)_{J^{c}}:=\left(x_{i_{1}}+\frac{d}{2}\left(r-i_{1}\right), \ldots, x_{i_{r-l}}+\frac{d}{2}\left(r-i_{r-l}\right)\right)_{i_{1}, \ldots, i_{r-l} \in J^{c}} .
$$

Originally, Theorem 1.2 or Corollary 3.1 were proved by Knop-Sahi [3]. Knop-Sahi's proof shows that $D_{r}^{(d) \text { ip }}(u ; \mathbf{x}) P_{\mathbf{k}}^{\text {ip }}\left(\mathbf{x}+\frac{d}{2} \boldsymbol{\delta} ; \frac{d}{2}\right)$ satisfy the conditions $(1)^{\text {ip }}$ and $(2)^{\text {ip }}$ up to a constant $c(\mathbf{k})$ for any $\mathbf{k} \in \mathcal{P}$, and determine $c(\mathbf{k})\left(=I_{r}^{(d)}(u ; \mathbf{k})\right)$ explicitly. Knop-Sahi's proof requires that the explicit expression of the difference operator $D_{r}^{(d) \text { ip }}(u ; \mathbf{x})$ for interpolation Jack polynomials are known in ad hoc, whereas our proof does not require it. 


\section{Pieri formulas for interpolation Jack polynomials}

Proof of Theorem 1.3. As with the proof of Theorem 1.2, it is enough to prove (1.9) for $\mathbf{x} \in \mathcal{P}$. For the purpose, we compute

$$
\left[e^{\operatorname{ad}\left|\partial_{\mathbf{z}}\right|} S_{r}^{(d)}(u ; \mathbf{z})\right] \Phi_{\mathbf{x}}^{(d)}(\mathbf{1}+\mathbf{z})=e^{\left|\partial_{\mathbf{z}}\right|} S_{r}^{(d)}(u ; \mathbf{z}) e^{-\left|\partial_{\mathbf{z}}\right|} \Phi_{\mathbf{x}}^{(d)}(\mathbf{1}+\mathbf{z})
$$

in two different ways. From the binomial (1.5) and the twisted Pieri (1.6)

$$
\begin{aligned}
{\left[e^{\operatorname{ad}\left|\partial_{\mathbf{z}}\right|} S_{r}^{(d)}(u ; \mathbf{z})\right] \Phi_{\mathbf{x}}^{(d)}(\mathbf{1}+\mathbf{z}) } & \\
= & \sum_{\mathbf{k} \subseteq \mathbf{x}} \frac{P_{\mathbf{k}}^{\mathrm{ip}}\left(\mathbf{x}+\frac{d}{2} \boldsymbol{\delta} ; \frac{d}{2}\right)}{P_{\mathbf{k}}\left(\mathbf{1} ; \frac{d}{2}\right)} \sum_{l=0}^{r}\left[\frac{\left(\operatorname{ad}\left|\partial_{\mathbf{z}}\right|\right)^{l}}{l !} S_{r}^{(d)}(u ; \mathbf{z})\right] \Psi_{\mathbf{k}}^{(d)}(\mathbf{z}) \\
& =\sum_{\mathbf{k} \subseteq \mathbf{x}} \frac{P_{\mathbf{k}}^{\mathrm{ip}}\left(\mathbf{x}+\frac{d}{2} \boldsymbol{\delta} ; \frac{d}{2}\right)}{P_{\mathbf{k}}\left(\mathbf{1} ; \frac{d}{2}\right)} \sum_{l=0}^{r} \sum_{\substack{\subseteq[r],|J|=l, \mathbf{k}-\epsilon_{J} \in \mathcal{P}}} \Psi_{\mathbf{k}-\epsilon_{J}}^{(d)}(\mathbf{z}) I_{J c}^{(d)}(u ; \mathbf{k}) h_{+, J}^{(d)}\left(\mathbf{k}-\epsilon_{J}\right) \\
& =\sum_{\mathbf{k} \in \mathcal{P}} \Psi_{\mathbf{k}}^{(d)}(\mathbf{z}) \sum_{\substack{J \subseteq[r], \mathbf{k}+\epsilon_{J} \in \mathcal{P}}} \frac{P_{\mathbf{k}}^{\mathrm{ip}}\left(\mathbf{x}+\frac{d}{2} \boldsymbol{\delta} ; \frac{d}{2}\right)}{P_{\mathbf{k}+\epsilon_{J}}\left(\mathbf{1} ; \frac{d}{2}\right)} I_{J^{c}}^{(d)}(u ; \mathbf{k}) A_{+, J}^{(d)}(\mathbf{k}) .
\end{aligned}
$$

The third equality follows from $I_{J^{c}}^{(d)}\left(u ; \mathbf{k}+\epsilon_{J}\right)=I_{J^{c}}^{(d)}(u ; \mathbf{k})$.

On the other hand, from (1.1) and the binomial formula (1.5),

$$
\begin{aligned}
{\left[e^{\mathrm{ad}\left|\partial_{\mathbf{z}}\right|} S_{r}^{(d)}(u ; \mathbf{z})\right] \Phi_{\mathbf{x}}^{(d)}(\mathbf{1}+\mathbf{z}) } & =e^{\left|\partial_{\mathbf{z}}\right|} S_{r}^{(d)}(u ; \mathbf{z}) e^{-\left|\partial_{\mathbf{z}}\right|} \Phi_{\mathbf{x}}^{(d)}(\mathbf{1}+\mathbf{z})=e^{\left|\partial_{\mathbf{z}}\right|} S_{r}^{(d)}(u ; \mathbf{z}) \Phi_{\mathbf{x}}^{(d)}(\mathbf{z}) \\
& =e^{\left|\partial_{\mathbf{z}}\right|} \Phi_{\mathbf{x}}^{(d)}(\mathbf{z}) I_{r}^{(d)}(u ; \mathbf{x})=\Phi_{\mathbf{x}}^{(d)}(\mathbf{1}+\mathbf{z}) I_{r}^{(d)}(u ; \mathbf{x}) \\
& =\sum_{\mathbf{k} \subseteq \mathbf{x}} \frac{P_{\mathbf{k}}^{\mathrm{ip}}\left(\mathbf{x}+\frac{d}{2} \boldsymbol{\delta} ; \frac{d}{2}\right)}{P_{\mathbf{k}}\left(\mathbf{1} ; \frac{d}{2}\right)} \Psi_{\mathbf{k}}^{(d)}(\mathbf{z}) I_{r}^{(d)}(u ; \mathbf{x}) \\
& =\sum_{\mathbf{k} \in \mathcal{P}} \Psi_{\mathbf{k}}^{(d)}(\mathbf{z}) I_{r}^{(d)}(u ; \mathbf{x}) \frac{P_{\mathbf{k}}^{\mathrm{ip}}\left(\mathbf{x}+\frac{d}{2} \boldsymbol{\delta} ; \frac{d}{2}\right)}{P_{\mathbf{k}}\left(\mathbf{1} ; \frac{d}{2}\right)} .
\end{aligned}
$$

By comparing coefficients for $u^{r-l}$ in (1.9), we obtain the Pieri type formulas for the interpolation Jack polynomials, which are a higher order analogue of equation (5.3) in [10] or equation $(14.2)$ in [5].

Corollary 4.1. For any $\mathbf{x} \in \mathbb{C}^{r}, \mathbf{k} \in \mathcal{P}$ and $l=0,1, \ldots, r$,

$$
\begin{aligned}
e_{r, l}\left(\mathbf{x}+\frac{d}{2} \boldsymbol{\delta}\right) \frac{P_{\mathbf{k}}^{\mathrm{ip}}\left(\mathbf{x}+\frac{d}{2} \boldsymbol{\delta} ; \frac{d}{2}\right)}{P_{\mathbf{k}}\left(\mathbf{1} ; \frac{d}{2}\right)} \\
\quad=\sum_{\substack{J \subseteq[r],|J|=l, \mathbf{k}+\epsilon_{J} \in \mathcal{P}}} \frac{P_{\mathbf{k}+\epsilon_{J}}^{\mathrm{ip}}\left(\mathbf{x}+\frac{d}{2} \boldsymbol{\delta} ; \frac{d}{2}\right)}{P_{\mathbf{k}+\epsilon_{J}}\left(\mathbf{1} ; \frac{d}{2}\right)} e_{r-|J|, l-|J|}\left(\left(\mathbf{k}+\frac{d}{2} \boldsymbol{\delta}\right)_{J^{c}}\right) A_{+, J}^{(d)}(\mathbf{k}) .
\end{aligned}
$$

\section{Some intertwining relations for the kernel function ${ }_{0} \mathcal{F}_{0}{ }^{(d)}(\mathrm{z}, \mathrm{w})$}

By comparing the coefficients for $u^{r-l}$ of the twisted Pieri formulas (1.6) and (1.7), we obtain the following twisted Pieri type formulas. 
Corollary 5.1. For any $\mathbf{z} \in \mathbb{C}^{r}$ and $l=0,1, \ldots, r$, we have

$$
\begin{aligned}
& \left(\frac{d}{2}\right)^{l}\left[\frac{\left(\operatorname{ad}\left|\partial_{\mathbf{z}}\right|\right)^{l}}{l !} H_{r, l}^{(d)}(\mathbf{z})\right] \Phi_{\mathbf{x}}^{(d)}(\mathbf{z})=\sum_{\substack{J \subseteq[r],|J|=l, \mathbf{x}-\epsilon_{J} \in \mathcal{P}}} \Phi_{\mathbf{x}-\epsilon_{J}}^{(d)}(\mathbf{z}) A_{-, J}^{(d)}(\mathbf{x}) \prod_{j \in J}\left(x_{j}+\frac{d}{2}(r-j)\right), \\
& \left(\frac{d}{2}\right)^{l}\left[\frac{\left(\operatorname{ad}\left|\partial_{\mathbf{z}}\right|\right)^{l}}{l !} H_{r, l}^{(d)}(\mathbf{z})\right] \Psi_{\mathbf{x}}^{(d)}(\mathbf{z})=\sum_{\substack{J \subseteq[r],|J|=l, \mathbf{x}-\epsilon_{J} \in \mathcal{P}}} \Psi_{\mathbf{x}-\epsilon_{J}}^{(d)}(\mathbf{z}) A_{+, J}^{(d)}\left(\mathbf{x}-\epsilon_{J}\right) .
\end{aligned}
$$

On the other hand, by comparing the coefficients for the highest degree term of (4.1) and the condition $(2)^{\text {ip }}$, we obtain the following well-known Pieri formulas for the ordinary Jack polynomials.

Corollary 5.2 (Macdonald [7], Stanley [16]). For $l=0,1, \ldots, r$,

$$
e_{r, l}(\mathbf{z}) \Phi_{\mathbf{k}}^{(d)}(\mathbf{z})=\sum_{\substack{J \subseteq[r],|J|=l, \mathbf{k}+\epsilon_{J} \in \mathcal{P}}} \Phi_{\mathbf{k}+\epsilon_{J}}^{(d)}(\mathbf{z}) A_{+, J}^{(d)}(\mathbf{k}) .
$$

From two Pieri type formulas (5.2) and (5.3), we prove Theorem 1.4 immediately. In fact, we have

$$
\begin{aligned}
\left(\frac{d}{2}\right)^{l}\left[\frac{\left(\operatorname{ad}\left|\partial_{\mathbf{z}}\right|\right)^{l}}{l !} H_{r, l}^{(d)}(\mathbf{z})\right]{ }_{0} \mathcal{F}_{0}{ }^{(d)}(\mathbf{z}, \mathbf{w}) & =\sum_{\mathbf{m} \in \mathcal{P}}\left(\frac{d}{2}\right)^{l}\left[\frac{\left(\operatorname{ad}\left|\partial_{\mathbf{z}}\right|\right)^{l}}{l !} H_{r, l}^{(d)}(\mathbf{z})\right] \Psi_{\mathbf{m}}^{(d)}(\mathbf{z}) \Phi_{\mathbf{m}}^{(d)}(\mathbf{w}) \\
& =\sum_{\mathbf{m} \in \mathcal{P}} \sum_{\substack{J \subseteq[r],|J|=l, \mathbf{m}-\epsilon_{J} \in \mathcal{P}}} \Psi_{\mathbf{m}-\epsilon_{J}}^{(d)}(\mathbf{z}) A_{+, J}^{(d)}\left(\mathbf{m}-\epsilon_{J}\right) \Phi_{\mathbf{m}}^{(d)}(\mathbf{w}) \\
& =\sum_{\mathbf{m} \in \mathcal{P}} \Psi_{\mathbf{m}}^{(d)}(\mathbf{z}) \sum_{\substack{J \subseteq[r],|J|=l, \mathbf{m}+\epsilon_{J} \in \mathcal{P}}} \Phi_{\mathbf{m}+\epsilon_{J}}^{(d)}(\mathbf{w}) A_{+, J}^{(d)}(\mathbf{m}) \\
& =\sum_{\mathbf{m} \in \mathcal{P}} \Psi_{\mathbf{m}}^{(d)}(\mathbf{z}) e_{r, l}(\mathbf{w}) \Phi_{\mathbf{m}}^{(d)}(\mathbf{w})={ }_{0} \mathcal{F}_{0}{ }^{(d)}(\mathbf{z}, \mathbf{w}) e_{r, l}(\mathbf{w}) .
\end{aligned}
$$

\section{Concluding remarks}

In this article, we have demonstrated usefulness of the twisted Pieri formulas (1.6) and (1.7). In fact, from (1.6) and (1.7), difference equations and Pieri formulas for interpolation Jack polynomials are derived in parallel. Further, we also obtain some intertwining relations for ${ }_{0} \mathcal{F}_{0}{ }^{(d)}(\mathbf{z}, \mathbf{w})$, which means that the twisted Pieri formulas (5.1) and (5.2) are dual of the usual Pieri formulas (5.3).

All our results in this paper are of formulas associated with $A$-type root system. Therefore it would be interesting to generalize twisted Pieri formulas from type $A$ to type $B C$. Some extension to $q, t$-analogue of our theorems is also an important problem. Sahi [14] introduced interpolation Macdonald polynomials which are $q, t$-analogue of interpolation Jack polynomials, and Okounkov [8] gave "Idea of Proof" of difference equations for interpolation Macdonald polynomials. Another proof of difference equations for interpolation Macdonald polynomials in our approach would be desirable. It would also be a challenging problem to explore a new method which is applicable further to the elliptic case $[1,12]$. 


\section{Acknowledgements}

We are grateful to Professor Masatoshi Noumi (Kobe University) for his helpful advice on our paper. We also wish to thank Professor Farrokh Atai for his valuable suggestions on Jack and interpolation Jack polynomials. Further, we thank the referees for their helpful comments. This work was supported by Grant-in-Aid for JSPS Fellows (Number 18J00233).

\section{References}

[1] Coskun H., Gustafson R.A., Well-poised Macdonald functions $W_{\lambda}$ and Jackson coefficients $\omega_{\lambda}$ on $B C_{n}$, in Jack, Hall-Littlewood and Macdonald polynomials, Contemp. Math., Vol. 417, Amer. Math. Soc., Providence, RI, 2006, 127-155, arXiv:math.CO/0412153.

[2] Debiard A., Polynômes de Tchébychev et de Jacobi dans un espace euclidien de dimension $p, C$. R. Acad. Sci. Paris Sér. I Math. 296 (1983), 529-532.

[3] Knop F., Sahi S., Difference equations and symmetric polynomials defined by their zeros, Int. Math. Res. Not. 1996 (1996), 473-486, arXiv:q-alg/9610017.

[4] Koornwinder T.H., Okounkov's BC-type interpolation Macdonald polynomials and their $q=1$ limit, Sém. Lothar. Combin. 72 (2015), Art. B72a, 27 pages, arXiv:1408.5993.

[5] Lassalle M., Coefficients binomiaux généralisés et polynômes de Macdonald, J. Funct. Anal. 158 (1998), $289-324$.

[6] Macdonald I.G., Schur functions: theme and variations, in Séminaire Lotharingien de Combinatoire (SaintNabor, 1992), Publ. Inst. Rech. Math. Av., Vol. 498, University Louis Pasteur, Strasbourg, 1992, 5-39.

[7] Macdonald I.G., Symmetric functions and Hall polynomials, 2nd ed., Oxford Mathematical Monographs, The Clarendon Press, Oxford University Press, New York, 1995.

[8] Okounkov A., Binomial formula for Macdonald polynomials and applications, Math. Res. Lett. 4 (1997), 533-553, arXiv:q-alg/9608021.

[9] Okounkov A., BC-type interpolation Macdonald polynomials and binomial formula for Koornwinder polynomials, Transform. Groups 3 (1998), 181-207, arXiv:q-alg/9611011.

[10] Okounkov A., Olshanski G., Shifted Jack polynomials, binomial formula, and applications, Math. Res. Lett. 4 (1997), 69-78, arXiv:q-alg/9608020.

[11] Okounkov A., Olshanski G., Shifted Schur functions, St. Petersburg Math. J. 9 (1998), 239-300, arXiv:q$\operatorname{alg} / 9605042$.

[12] Rains E.M., $B C_{n}$-symmetric Abelian functions, Duke Math. J. $135 \quad$ (2006), 99-180, arXiv:math.CO/0402113.

[13] Sahi S., The spectrum of certain invariant differential operators associated to a Hermitian symmetric space, in Lie Theory and Geometry, Progr. Math., Vol. 123, Birkhäuser Boston, Boston, MA, 1994, 569-576.

[14] Sahi S., Interpolation, integrality, and a generalization of Macdonald's polynomials, Int. Math. Res. Not. 1996 (1996), 457-471.

[15] Sekiguchi J., Zonal spherical functions on some symmetric spaces, Publ. Res. Inst. Math. Sci. 12 (1977), 455-459.

[16] Stanley R.P., Some combinatorial properties of Jack symmetric functions, Adv. Math. 77 (1989), $76-115$.

[17] Vilenkin N.Ja., Klimyk A.U., Representation of Lie groups and special functions. Recent advances, Mathematics and its Applications, Vol. 316, Kluwer Academic Publishers Group, Dordrecht, 1995. 